\title{
OTIMIZAÇÃO DE PRÉ-TRATAMENTO ÁCIDO DO BAGAÇO DE CANA-DE-AÇÚCAR PARA OBTENÇÃO DE AÇÚCARES FERMENTESCÍVEIS
}

\author{
E. L. SCHULTZ, A. RODRIGUES, T. D. MENDES, L. M. C. FILARDI, R. B. \\ CAMPANHA, T. F. PACHECO, D. S. R. GAMBETTA, C. M. RODRIGUES, M. C. \\ T. DAMASO \\ Embrapa Agroenergia \\ E-mail para contato: emerson.schultz@embrapa.br
}

\begin{abstract}
RESUMO - Materiais lignocelulósicos contêm polissacarídeos que podem ser convertidos em açúcares fermentescíveis para obtenção de Etanol 2G e outros bioprodutos. Para essa conversão, são necessários processos de pré-tratamento e hidrólise enzimática eficientes e eficazes. O objetivo deste trabalho foi otimizar as condições de pré-tratamento ácido de bagaço de cana-de-açúcar visando obter maiores quantidades destes açúcares. As condições experimentais do pré-tratamento foram estabelecidas por um planejamento DCCR $2^{2}$, variando as concentrações de ácido sulfúrico $(0,1$ a $2,9 \%)$ e os tempos de reação (14 a $56 \mathrm{~min})$, a $120^{\circ} \mathrm{C}$ e com razão sólido/líquido de 1:10. Utilizando esta mesma razão, a celulignina foi hidrolisada com Cellic ${ }^{\circledR}$ CTec 3 (Novozymes), considerando $15 \mathrm{FPU} / \mathrm{g}$ de biomassa, a $50^{\circ} \mathrm{C}$ por $48 \mathrm{~h}$. Amostras foram retiradas durante este intervalo e os açúcares quantificados por HPLCRID. O melhor resultado de pré-tratamento, em termos de glicose gerada, foi alcançado com 1,5\% de ácido e 56 min, sendo que foram gerados 24,7 e 29,0 g/L após 24 e $48 \mathrm{~h}$, respectivamente.Após $24 \mathrm{~h}$ de hidrólise, foram obtidos resultados de conversão da celulose disponível entre 26,9 e $42,1 \%$. A análise estatística dos dados mostrou que todas as variáveis, inclusive as interações entre elas, são significativas para a obtenção de glicose. Este mesmo perfil foi verificado para o modelo predito, sendo possível identificar a região de ótimo de conversão.
\end{abstract}

\section{INTRODUÇÃO}

O desenvolvimento de produtos a partir de biomassa lignocelulósica é uma das áreas de pesquisa tecnológica que tem atraído amplo interesse. Diversos materiais lignocelulósicos podem ser usados para produção de etanol, sendo que o bagaço de cana-de-açúcar é um dos mais promissores (Cardonaet al., 2010; Betancur e Pereira Jr., 2010). Os processos empregados para produção de etanol a partir desses materiais incluem: trituração, prétratamento, hidrólise enzimática, fermentação e separação/purificação (Mussatoet al.; 2010).

A biomassa lignocelulósica possui excelentes mecanismos para proteger seus carboidratos estruturais da degradação por microrganismos e enzimas. Os processos de prétratamento da biomassa alteram seus fatores estruturais (volume e tamanho dos poros, tamanho da partícula e área superficial específica), sua composição química (lignina, 
hemiceluloses e grupo acetil) e fatores da estrutura da celulose (cristalinidade e grau de polimerização). Além disso, o pré-tratamento aumenta a área superficial, melhorando a acessibilidade da celulose pelas enzimas na etapa de hidrólise enzimática (Zhaoet al., 2012).

Entre os processos de pré-tratamento, a hidrólise com ácido diluído é um dos mais estudados. Esse pré-tratamento pode ser realizado com diferentes ácidos, como ácido sulfúrico, ácido clorídrico, ácido acético e ácido fosfórico. A hidrólise com ácido diluído resulta na solubilização da hemicelulose, separando a biomassa em uma fração líquida contendo, predominantemente,pentoses e uma fração sólida composta, majoritariamente, por celulose e lignina (Mussatoet al.; 2010). Dependendo das condições operacionais, a fração líquida será constituída de açúcares (xilose, glicose e arabinose) e produtos da decomposição das hemiceluloses (como oligômeros dos polímeros e ácido acético gerado pela hidrólise de grupos acetil ligados aos açúcares) e/ou produtos da decomposição de monossacarídeos (como furfural, produto da desidratação de pentoses, e 5-hidroximetilfurfural (HMF), produto da desidratação de hexoses) (Gámezet al., 2006).

Entre as condições operacionais que afetam o pré-tratamento ácido estão concentração do ácido, tempo e temperatura. A variação nessas condições afeta a severidade do prétratamento e a composição da biomassa de modo diferente durante o processo. Além disso, a razão sólido/líquido também exerce influência no processo, tendo relação com a concentração de xilose e ácido acético na fração líquida (Pedersen e Meyer, 2010, Betancur e Pereira Jr., 2010). Em geral, a avaliação do pré-tratamento tem sido baseada no subsequente rendimento de monossacarídeos após a hidrólise enzimática (Pedersen e Meyer, 2010, Alviraet al., 2011).

Dessa forma, o objetivo deste trabalho foi otimizar as condições de pré-tratamento ácido de bagaço de cana-de-açúcar visando obter maiores quantidades de açúcares fermentescíveis após hidrólise enzimática.

\section{MATERIAL E MÉTODOS}

A metodologia adotada incluiu o pré-tratamento ácido do bagaço de cana-de-açúcar, e sua caracterização, hidrólise enzimática e análise do hidrolisado.

\subsection{Matéria-prima e reagentes}

O bagaço de cana-de-açúcar utilizado neste trabalho foi gentilmente cedido pela Usina Jalles Machado, localizada em Goianésia/Goiás, Brasil. A biomassa foi seca ao sol e previamente moída em moinho de martelos. O sólido obtido após o processo de prétratamento ácido, denominado celulignina, foi utilizado como matéria-prima para hidrólise enzimática. A celulignina é composta basicamente por celulose e lignina. Os reagentes utilizados no trabalho eram de grau analítico.

\subsection{Pré-tratamento}

Diferentes condições de pré-tratamento ácido foram testadas, variando-se o tempo dereação e a concentração de ácido sulfúrico, visando obter o maior rendimento na obtenção de glicose. As condições experimentais foram estabelecidas usando um planejamento experimental do tipo Delineamento Central Composto Rotacional - DCCR, incluindo 
4ensaios nas condições axiais e 3 repetições no ponto central. Os valores dos níveis e fatores utilizados no planejamento estão especificados na Tabela 1.

A cada 20 g de bagaço de cana-de-açúcar foram adicionadas soluções de ácido sulfúrico nas concentrações especificadas na Tabela 1, observando-se a razão sólido/líquido de 1:10. O pré-tratamento foi realizado em autoclave a $120^{\circ} \mathrm{C}$, nos diferentes tempos de reação. Após o pré-tratamento, a celulignina (fração sólida) foi filtrada, sendo separada da fração líquida (licor), rica em pentoses. O licor teve o seu $\mathrm{pH}$ determinado para o cálculo do fator de severidade combinado, utilizando o método descrito por Schellet al. (2003). A fração sólida foi lavada por 3 vezes, com $200 \mathrm{~mL}$ de água destilada em cada lavagem, e encaminhada para a hidrólise enzimática.

Tabela 1- Valores utilizados no DDCR para o pré-tratamento ácido de bagaço de cana-deaçúcar

\begin{tabular}{lccccc}
\cline { 2 - 6 } & \multicolumn{5}{c}{ Níveis } \\
\hline Fatores & $-\alpha$ & -1 & 0 & +1 & $+\alpha$ \\
Conc. Ácido (\%) & 0,1 & 0,5 & 1,5 & 2,5 & 2,9 \\
Tempo (min) & 14 & 20 & 35 & 50 & 56 \\
\hline
\end{tabular}

\subsection{Caracterização da celulignina e do licor}

Para a caracterização do bagaço de cana-de-açúcar pré-tratado foram utilizados protocolos propostos pelo NationalRenewable Energy Laboratory - NREL. A separação das frações sólidas e líquidas da biomassa pré-tratada foi feita de acordo com NREL/TP-51042627(Sluiter, 2008 b); o teor de matéria-seca foi quantificado conforme NREL/TP-51042621(Sluiter, 2008 a) e teor de glucanase xilanas segundo NREL/TP-510-42618(Sluiter, 2012). O licor gerado no pré-tratamento foi analisado em relação à presença de açúcares, ácido acético, hidroximetilfurfural e furfuralporcromatografia líquida (HPLCRID)empregando coluna de troca iônica (HPX-87H). Os valores de $\mathrm{pH}$ do licor foram medidos, possibilitando o cálculo do fator de severidade combinado (FSC) dos ensaios de prétratamento (Schellet al., 2003).

\subsection{Hidrólise enzimática}

A biomassa pré-tratada, ainda úmida, foi suspensa (na proporção 1:10, em base seca) em tampão citrato de sódio/ácido cítrico $0,1 \mathrm{M}, \mathrm{pH}$ 5. Para cada grama de celulignina (massa seca) foi adicionado o correspondente a 15 FPU do complexo enzimático Cellic ${ }^{\circledR}$ CTec3 (Novozymes). A hidrólise foi realizada em frascos Erlenmeyers de $250 \mathrm{~mL}$, em agitador orbital, sob agitação constante de $200 \mathrm{rpm}$, na temperatura de $50^{\circ} \mathrm{C}$ por 48 horas. Alíquotas foram retiradas após os tempos de reação de $0,2,4,6,8,24$ e 48h. As quantidades de glicose, xilose, celobiose e arabinosegeradas após hidrólise foram avaliadas por HPLC-RID. As alíquotas referentes aos tempos 24 e $48 \mathrm{~h}$ foram avaliadas, a partir do mesmo método, em relação à presença de inibidores. 


\section{RESULTADOS}

Após pré-tratamento e hidrólise enzimática foram determinados os valores de concentração de glicose gerada e conversão de celulose (glucanas) em glicose, nos tempos de hidrólise enzimática de 24 e $48 \mathrm{~h}$. Os resultados obtidos podem ser visualizados na Tabela 2.

Tabela 2 - Matriz do delineamento e respostas de glicose gerada e conversão de celulose em glicose após 24 e $48 \mathrm{~h}$ de hidrólise enzimática

\begin{tabular}{ccccccc}
\hline Ensaios & $\begin{array}{c}\text { Tempo } \\
(\mathrm{min})\end{array}$ & $\begin{array}{c}{\left[\mathrm{H}_{2} \mathrm{SO}_{4}\right]} \\
(\% \text { em vol. })\end{array}$ & $\begin{array}{c}\text { [Glicose] } \mathrm{g} / \mathrm{L} \\
24 \mathrm{~h}\end{array}$ & $\begin{array}{c}{[\text { Glicose] } \mathrm{g} / \mathrm{L}} \\
48 \mathrm{~h}\end{array}$ & $\begin{array}{c}\text { Conversão } \\
(\%) 24 \mathrm{~h}\end{array}$ & $\begin{array}{c}\text { Conversão } \\
(\%) 48 \mathrm{~h}\end{array}$ \\
\hline 1 & 20 & 0,5 & 12,8 & 13,8 & 27,4 & 29,5 \\
2 & 20 & 2,5 & 19,5 & 22,0 & 36,3 & 41,0 \\
3 & 50 & 0,5 & 19,8 & 22,2 & 39,0 & 43,8 \\
4 & 50 & 2,5 & 23,5 & 27,5 & 39,9 & 46,7 \\
5 & 35 & 1,5 & 21,6 & 23,8 & 39,9 & 44,0 \\
6 & 35 & 1,5 & 21,7 & 23,7 & 40,0 & 43,8 \\
7 & 35 & 1,5 & 23,2 & 27,6 & 41,0 & 48,9 \\
8 & 35 & 0,1 & 11,3 & 13,3 & 26,9 & 31,8 \\
9 & 35 & 2,9 & 21,7 & 25,4 & 37,5 & 43,8 \\
10 & 14 & 1,5 & 17,1 & 20,9 & 32,8 & 40,2 \\
11 & 56 & 1,5 & 24,7 & 29,0 & 42,1 & 49,6 \\
\hline
\end{tabular}

Nas condições de ensaio realizadas, os maiores valores obtidos tanto para concentração de glicose gerada, como para conversão de celulose em glicose, foram obtidos no ensaio 11, com a condição de processo de 1,5\% de ácido sulfúrico e 56 min de reação, correspondentes a condição de maior tempo de reação e condição central da concentração de ácido. Os menores valores para as variáveis de resposta foram obtidos nas condições de menor concentração do ácido, tanto para tempos menores de reação (ensaio 1) como também para a condição central (ensaio 8). Não foi detectada a presença dos inibidores: ácido acético, furfural e hidroximetilfurfural nos hidrolisados enzimáticos obtidos após 24 e $48 \mathrm{~h}$ de hidrólise (dados não apresentados). O hidrolisado obtido na melhor condição de pré-tratamento continha 2,5g/L de xilose e não foi detectada presença de arabinose, e nem de celobiose, provavelmente, pela alta eficiência da conversão de celobioseem glicose.

Os licores obtidos em todos os ensaios foram analisados em relação à geração de possíveis inibidores formados durante a etapa de pré-tratamento do bagaço de cana-de-açúcar. A presença de ácido acético, furfural e hidroximetilfurfuralfoi avaliada (dados não apresentados). Paralelamente, foi medido o valor do $\mathrm{pH}$ do licor gerado em cada ensaio. Com base neste resultado foi possível calcular o fator de severidade combinado (FSC) para todas as condições de pré-tratamento, os quais variaram de 0,40 a 1,77. Schellet al. (2003) encontraram valores de FSC entre 0,57 e 2,02 para o pré-tratamento ácido de resíduos de milho. Fatores de severidade mais elevados podem indicar maior grau de solubilização da hemicelulose de biomassa utilizada (Pedersen e Meyer, 2010), o que foi constatado nos resultados obtidos no presente trabalho para os licores oriundos dos diferentes ensaios. 
A presença de furfural e de hidroximetilfurfural nos licores foi praticamente desprezível, enquanto que ácido acético foi detectado em concentrações que variaram entre 0,27 e 3,66 g/L. Betancur e Pereira Jr. (2010) também encontraram maiores concentrações de ácido acético $(4,42$ a $11,00 \mathrm{~g} / \mathrm{L})$ do que furfural $(0,31$ a $2,25 \mathrm{~g} / \mathrm{L})$ e hidroximetilfurfural $(0,05$ a $0,12 \mathrm{~g} / \mathrm{L})$ no licor obtido no pré-tratamento com ácido sulfúrico de bagaço de cana-deaçúcar. Os maiores valores encontrados por esses autores para concentração de ácido acético podem estar relacionados com a maior razão sólido/líquido empregada no pré-tratamento. Analisando-se os resultados do presente trabalho, foi possível detectar uma tendência de aumento da quantidade de ácido formado com o aumento do fator de severidade. Porém esta concentração não parece ter afetado a hidrólise enzimática, pois o ensaio 11 (Tabela 2), que apresentou o melhor resultado em termos das variáveis resposta possui um dos maiores fatores de severidade $(1,56)$.

Para avaliar o efeito do pré-tratamento, a análise estatística dos resultados foi realizada somente para a variável resposta conversão de celulose em glicose, após $24 \mathrm{~h}$ de hidrólise. Esta escolha ocorreu em virtude dos melhores resultados terem sido obtidos neste tempo de reação, levando-se em consideração questões de produtividade. A Figura 1 mostra o Gráfico de Pareto, que ilustra de forma simplificada o efeito das variáveis: tempo de reação e concentração de ácido no pré-tratamento sob a conversão de celulose em glicose, na hidrólise enzimática. Com base nos resultados apresentados, foi possível verificar que tanto os termos lineares quanto os quadráticos e a interação entre as variáveis: tempo de reação e concentração de ácido foram estatisticamente significativas ( $<<0,05)$, na faixa avaliada (Teste t). Os efeitos lineares das variáveis: tempo de reação e concentração de ácido indicaram que o aumento destas variáveis acarretou um aumento na variável resposta. Os efeitos quadráticos e a interação entre os fatores indicou um comportamento oposto (Figura 1).

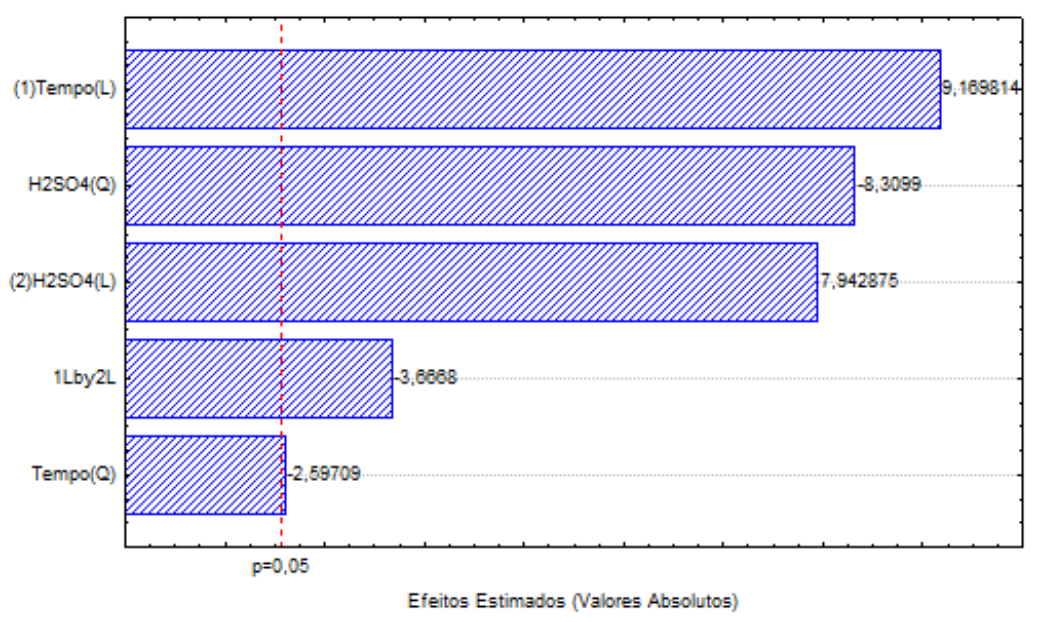

Figura 1- Gráfico de Pareto dos efeitos estimados (valores absolutos) do tempo de reação e concentração de ácido sobre a conversão da celulose (\%) em 24 horas de reação.

Com os resultados gerados foi possível determinar os coeficientes de regressão, e como todos os fatores, inclusive a interação entre eles foi significativa para a variável resposta, todos os coeficientes podem ser inseridos para elaborar um modelo com as variáveis codificadas, conforme apresentado na Equação 1. A letra t indica o tempo de reação e a letra 
c, a concentração do ácido.

$$
\text { Conversão(\%)=39,874+3,526t-1,192 } t^{2}+3,054 c-3,813 c^{2}-1,991 t c
$$

Com base no resultado do teste ANOVA apresentado na Tabela 3, observa-se que o modelo que descreve a conversão de celulose em glicose, após $24 \mathrm{~h}$ de hidrólise é adequado, visto que a percentagem de variação explicada é de $98 \%$ e o $\mathrm{F}$ calculado é altamente significativo, satisfazendo os requisitos para a construção das superfícies de resposta (Figura 2) e validação do modelo predito (Equação 1) com variáveis descodificadas.

Tabela 3. ANOVA para a resposta conversão de celulose em glicose após $24 \mathrm{~h}$ de hidrólise enzimática

\begin{tabular}{lcccc}
\hline \multicolumn{1}{c}{ Fonte de } & Soma dos & Graus de & Quadrado & $\mathrm{F}_{\text {calc }}$ \\
variação & Quadrados & liberdade & Médio F & \\
\hline Regressão & 278,80 & 5 & 55,76 & 47,3 \\
$\begin{array}{l}\text { Resíduos } \\
\text { Total }\end{array}$ & 5,90 & 5 & 1,18 & \\
$\%$ variação explicada $\left(\mathrm{R}^{2}=97,9\right) ; \mathrm{F}_{5,5}=5,05$ & & \\
\hline
\end{tabular}

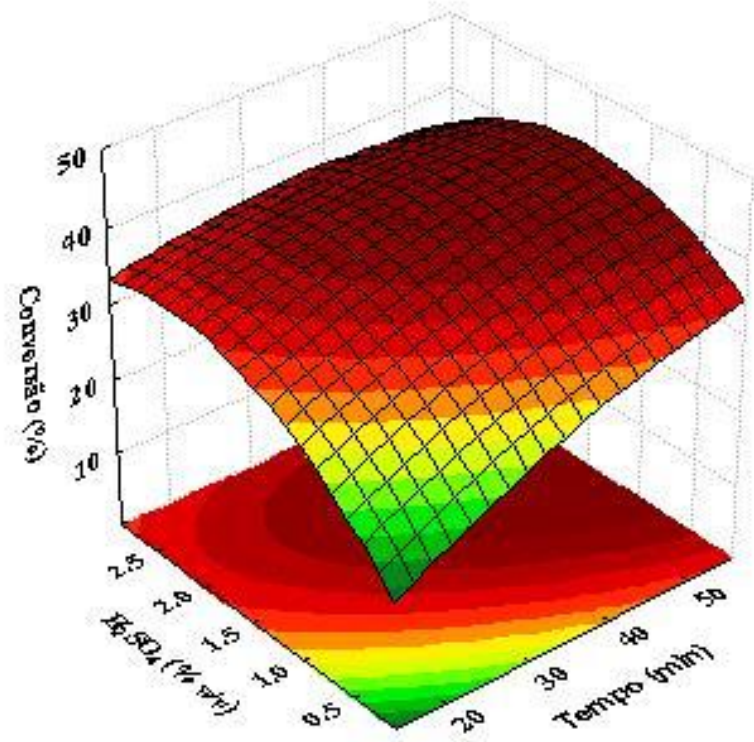

Figura 2- Superfície de resposta da conversão de celulose em glicose após 24 h de hidrólise enzimática, em função do tempo de reação e da concentração de ácido.

Com base na superfície de resposta (Figura 2) pode ser definida a região de interesse de maior conversão, dentro da faixa estudada. Verificou-se que a região de ótimo se encontra nos tempos de reação maiores e nas concentrações de ácido próximas do ponto central. Para 
validação dos resultados obtidos foram escolhidas 3 condições de processo, dentro da região de ótimo, a saber: 39,5 min e $1,9 \% \mathrm{H}_{2} \mathrm{SO}_{4} ; 53$ min e $1,6 \% \mathrm{H}_{2} \mathrm{SO}_{4} ; 56$ min e $1,1 \% \mathrm{H}_{2} \mathrm{SO}_{4}$.

Na Figura 3 estão apresentados os perfis cinéticos da hidrólise enzimática das 3 condições selecionadas. Verificou-se que houve um incremento na taxa de conversão de no máximo $12 \%$ entre 24 e $48 \mathrm{~h}$ de hidrólise, indicando que se fosse realizada uma análise técnica e econômica do processo, provavelmente, o tempo de $24 \mathrm{~h}$ seria o escolhido para hidrólise enzimática.

Os dados utilizados para construção do gráfico são as médias de triplicatas, com seus desvios padrão. Baixos valores de desvio padrão foram encontrados, o que indicou uma ótima reprodutibilidade dos dados. Além disso, os valores de conversão foram idênticos nas 3 condições testadas, corroborando com os resultados encontrados pela análise estatística dos dados, que previu alta significância do modelo predito.

No entanto, os resultados de conversão, após $24 \mathrm{~h}$ de hidrólise, obtidos nestes 3 pontos selecionados foram, no máximo, $15 \%$ superiores aos esperados pelo modelo. Como o cálculo da conversão depende tanto da caracterização da celulignina, quanto da glicose quantificada após hidrólise, acredita-se que este aumento tenha ocorrido por questões inerentes às metodologias utilizadas em ambos os processos.

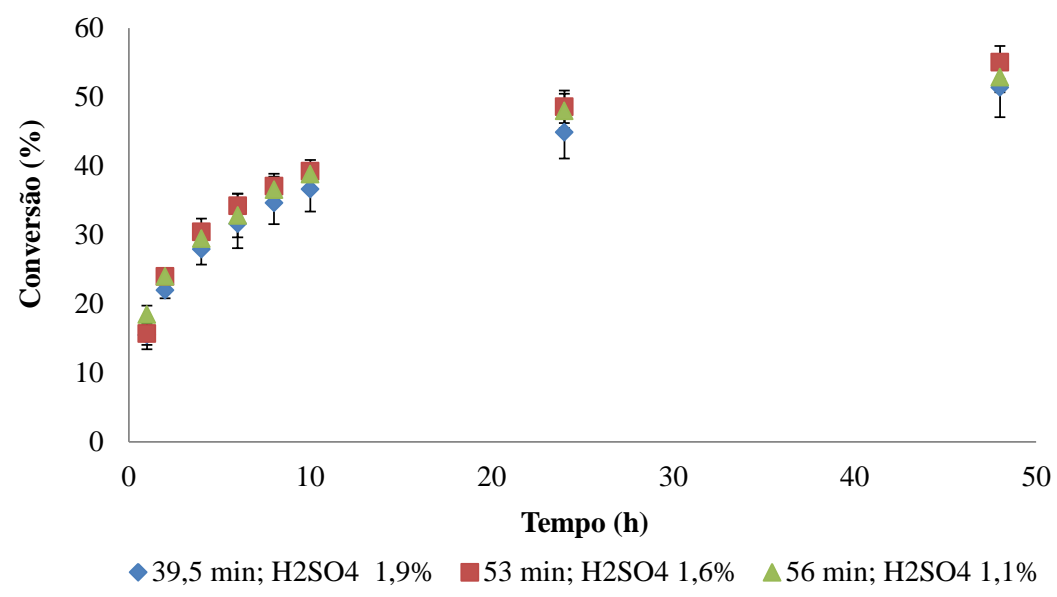

Figura 3- Perfil cinético da hidrólise enzimática de 3 condições de processo selecionadas da região de ótimo de conversão.

\section{CONCLUSÕES}

O melhor resultado, em termos de glicose, foi alcançado com 1,5\% de ácido e 56 min: 24,7 e 29,0 g/L após 24 e 48 h, respectivamente.Após 24 h de hidrólise, foram obtidos resultados de conversão da celulose disponível entre 26,9 e 42,1\%. As variáveis estudadas, inclusive a interação entre elas, foram estatisticamente significativas. O modelo predito foi estatisticamente significativo, sendo possível identificar a região de ótimo de conversão, a qual se encontra nos tempos de reação maiores e nas concentrações de ácido próximas do ponto central. 
A presença de furfural e de hidroximetilfurfural nos licores foi praticamente desprezível, enquanto ácido acético foi detectado em concentrações que variaram entre 0,27 e 3,66 g/L. Foi verificadauma tendência de aumento da quantidade de ácido formado com o aumento do fator de severidade combinado nas condições de pré-tratamento testadas, no entanto,não houve a indicação que a presença do ácido tenha influenciado a hidrólise enzimática.

\section{REFERÊNCIAS}

ALVIRA, P.; TOMÀS-PEJÓ, E.; BALLESTEROS, M.; NEGRO, M. J. Pretreatment technologies for an efficient bioethanol production process based on enzymatyc hydrolysis: A review. Bioresour.Technol., v. 101, p. 4851-4861, 2010.

BETANCUR, G. J. V.; PEREIRA JR. N. Sugar cane bagasse as feedstock for second generation etanol production. Part I: Diluted acid pretreatment optimization. Electron. J. Biotechn., v. 13, n. 3, 2010.

CARDONA, C. A.; QUINTERO, J. A.; PAZ, I. C. Production of bioethanol from sugarcane bagasse: Status and perspectives. Bioresour.Technol., v. 101, p. 4754-4766, 2010.

GÁMEZ, S.; GONZÁLEZ-CABRIALES， J. J.; RAMÍREZ， J. A.; GARROTE， G.; VÁZQUEZ, M. Study of the hydrolysis of sugar cane bagasse using phosphoric acid. $J$. Food Eng., v. 74, p. 78-88, 2006.

MUSSATO, S. I.; DRAGONE, G.; GUIMARÃES, P. M. R.; SILVA, J. P. A.; CARNEIRO, L. M.; ROBERTO, I. C.; VICENTE, A.; DOMINGUES, L; TEIXEIRA, J. A. Technological trends, global Market, and challenges of bio-ethanol production. Biotechnol.Adv., v. 28, p. 817-830, 2010.

PEDERSEN, M.; MEYER, A. S. Lignocellulose pretreatment severity - relating $\mathrm{pH}$ to biomatrix opening.New Biotechnol., v. 27, n. 6, p. 739-750, 2010.

SCHELL, D. J.; FARMER, J.; NEWMAN, M.; McMILLAN, J. D. Dilute-Sulfuric Acid Pretreatment of Corn Stover in Pilot-Scale Reactor.Appl. Biochem. Biotechnol., v. 105108, p. 69-85, 2003.

SLUITER, A.; HAMES, B.; HYMAN, D.; PAYNE, C.; RUIZ, R.; SCARLATA, C.; SLUITER, J.; TEMPLETON, D.; WOLFE, J. Determination of Total Solids in Biomass and Total Dissolved Solids in Liquid Process Samples.2008 a. In: NREL/TP-510-42621 - NREL - National Renewable Energy Laboratory. Technical Report.LaboratoryAnalytical Procedures (LAP). Disponível em <http://www.nrel.gov/publications/> Acesso em 15/04/2013.

SLUITER, A.; HAMES, B.; RUIZ, R.; SCARLATA, C.; SLUITER, J.; TEMPLETON, D.; CROCKER, D. Determination of Structural Carbohydrates and Lignin in Biomass. 2012. In: NREL/TP-510-42618 - NREL - National Renewable Energy Laboratory. Technical Report.LaboratoryAnalytical Procedures (LAP). Disponível em <http://www.nrel.gov/publications/> Acesso em 15/04/2013.

SLUITER, A.; HYMAN, D.; PAYNE, C.; WOLFE, J. Determination of Insoluble Solids in Pretreated Biomass Material.2008 b. In: NREL/TP-510-42627 - NREL - National Renewable Energy Laboratory. Technical Report.LaboratoryAnalytical Procedures (LAP). Disponível em <http://www.nrel.gov/publications/> Acesso em 15/04/2013.

ZHAO, X.; ZHANG, L.; LIU, D. Biomass recalcitrance. Part I: the chemical compositions and physical structures affecting the enzymatic hydrolysis of lignocellulose. Biofuels, Bioprod. Biorefin., v. 6, p. 465-482, 2012. 